Bundesgesundheitsbl 2015 - 58:182-189 DOI 10.1007/s00103-014-2098-x

Online publiziert: 5. Dezember 2014

c) Springer-Verlag Berlin Heidelberg 2014

\author{
S. Petersen ${ }^{1} \cdot$ H.F. Rabenau ${ }^{2} \cdot$ A. Mankertz ${ }^{3}$ D. Matysiak-Klose ${ }^{4} \cdot$ I. Friedrichs ${ }^{2}$. \\ S. Wicker ${ }^{1}$ \\ ${ }^{1}$ Betriebsärztlicher Dienst, Universitätsklinikum Frankfurt, Frankfurt am Main, Deutschland \\ 2 Institut für Medizinische Virologie, Universitätsklinikum Frankfurt, Frankfurt am Main, Deutschland \\ ${ }^{3}$ Nationales Referenzzentrum Masern, Mumps, Röteln, Robert Koch-Institut, Berlin, Deutschland \\ ${ }^{4}$ Geschäftsstelle der Nationalen Verifizierungskommission Masern/Röteln, \\ Fachgebiet Impfprävention, Robert Koch-Institut, Berlin, Deutschland
}

\title{
Immunität gegen Masern beim medizinischen Personal des Universitätsklinikums Frankfurt, 2003-2013
}

einmalige Impfung erhalten, 27\% wurden mindestens zweimal gegen die Masern geimpft. Für das Jahr 2014 ist eine Schwankung zu verzeichnen, bis zum 30.11.2014 gingen am RKI 364 Meldungen ein [7].

Insbesondere in Ländern in der Präeliminationsphase und mit sinkender Maserninzidenz spielt die nosokomiale Masernübertragung eine wichtige Rolle: Hier sind $14-45 \%$ der Infektionsfälle bei Masernepidemien nosokomialen Ursprungs $[8,9]$. Das berufliche Infektionsrisiko von medizinischem Personal wird 2- bis 19-mal so hoch wie das Infektionsrisiko der Normalbevölkerung geschätzt [1, $10,11]$. Aus diesem Grund ist der Masernschutz für medizinisches Personal unabdingbar $[1,12,13]$.

Die Impfeffektivität der zweimaligen Masernimpfung zur Verhinderung einer Masernerkrankung ist sehr hoch und wird mit 92-99\% angegeben [14]. Dennoch kann es auch bei Geimpften zur Infektion und zur Manifestation der Erkrankung kommen. Bislang ging man davon aus, dass geimpfte Personen mit einem Maserndurchbruch nicht ansteckend sind. Eine aktuelle Studie belegte jedoch, dass eine 2-mal MMR-geimpfte 22-Jährige vier Personen, die entweder 2-mal geimpft waren oder anamnestisch positive Masern IgG-Antikörper aufwiesen, angesteckt hatte. Alle Patienten zeigten eine klinische Masernsymptomatik, die Maserninfektion wurde laborbestä- tigt und ergab IgG-Antikörper mit hoher Avidität als Zeichen einer sekundären Immunantwort [15]. Bislang gelten diese Fälle als Ausnahmen. Daher werden serologische Untersuchungen zur Überprüfung des Impferfolgs von der Ständigen Impfkommission (STIKO) und dem Advisory Committee on Immunization Practices (ACIP) als nicht erforderlich erachtet $[12,16]$. Die zu erwartenden Auswirkungen absinkender Titer und eines nachlassenden natürlichen Boostereffektes müssen untersucht werden und sind ein zusätzliches Argument für die zügige Umsetzung der Masernelimination. Da ein primäres und sekundäres Versagen bei Masernimpfungen wiederholt beschrieben wurde, werden aufgrund des erhöhten Risikos für nosokomiale Infektionen am Universitätsklinikum Frankfurt beim medizinischen Personal und bei den Medizinstudierenden seit 2003 sowohl Impfpasskontrollen als auch serologische Untersuchungen zur Bestimmung der Masernimmunität durchgeführt.

Ziel unserer Studie war es, den Masernimmunstatus des medizinischen Personals und der Medizinstudierenden im Zeitverlauf zu ermitteln. Es sollte analysiert werden, ob entsprechend dem Maserneliminationsziel der WHO mehr als 95\% der Untersuchten eine positive Masernimmunität aufweisen. Darüber hinaus wurden das Wissen der Medizinstudierenden zum Maserneliminationsziel 
der WHO und die Akzeptanz der Impfempfehlungen überprüft, um die individuelle Bewertung der Krankheitslast und die Notwendigkeit der Implementierung von Schulungs- bzw. Public-Health-Maßnahmen beurteilen zu können.

\section{Methoden}

Das Universitätsklinikum Frankfurt am Main ist ein Klinikum der unabdingbaren Notfallversorgung mit 1187 Betten und 4105 Mitarbeitern. Im Studiengang Humanmedizin der Goethe-Universität waren im Sommersemester 2014 insgesamt 2809 Studierende immatrikuliert.

Seit 2003 wird im Rahmen der arbeitsmedizinischen Vorsorge u. a. der Masernserostatus des medizinischen Personals mit direktem Patientenkontakt und der Medizinstudierenden ab dem Ersten Abschnitt der Ärztlichen Prüfung (Physikum) bei Beginn der Tätigkeit bestimmt. Liegt ein positiver serologischer Masernbefund vor, erfolgen keine weiteren Untersuchungen. Arbeitsmedizinisch indizierte Impfungen werden angeboten und durchgeführt. Für die vorliegende Studie fand eine retrospektive Analyse der 9933 Ergebnisse von Masernimmunitätsbestimmungen der Mitarbeiter und Medizinstudierenden über den Zeitraum Januar 2003 bis Dezember 2013 statt.

Die Datenauswertung erfolgte anhand der Datenbank des Institutes für Medizinische Virologie auf Basis der einsenderbezogenen Datensätze des Betriebsärztlichen Dienstes. Die Angaben umfassen Alter, Geschlecht, Art der Beschäftigung (Personal oder Studierender) und den Masernserostatus mit Angabe des Testwertes. Da bei der Datenextraktion keine Namen der Getesteten extrahiert wurden, war eine namentliche Rückverfolgung nicht möglich.

\section{Serologische Testung}

Die Proben wurden am Institut für Medizinische Virologie des Universitätsklinikums Frankfurt untersucht. Während des gesamten Studienzeitraums erfolgte die Bestimmung der masernspezifischen IgGAntikörper automatisiert mittels kommerziell erhältlichen Testkits
(Enzygnost Anti-Masern-IgG, Fa. Siemens Healthcare Diagnostics GmbH, Eschborn, Deutschland) unter Verwendung eines BEP III und der sog. $\alpha-M-$ ethode. Zur Bewertung der Immunitätslage fand eine Einteilung in folgende Kategorien statt:

- positives Testergebnis: Immunität anzunehmen,

- grenzwertiges Testergebnis: Immunität fraglich,

- negatives Testergebnis: keine Immunität anzunehmen.

\section{Anonyme Fragebogenerhebung}

Ein für die Studie entwickelter standardisierter Fragebogen wurde von März 2014 bis Mai 2014 an die Medizinstudierenden der Goethe-Universität verteilt. Um möglichst viele Studierende aller Fachsemester zu erreichen, wurden die Fragebögen nach Hauptvorlesungen und Semesterabschlussklausuren und bei Erhalt der $\mathrm{Zu}$ lassung zur Zweiten Ärztlichen Prüfung sowie im PJ-Seminar verteilt.

Der Fragebogen wurde anhand ähnlicher Studien (Literaturrecherche in PubMed) entwickelt und in einer Vortestung mit 20 Medizinstudierenden evaluiert.

Der einseitige Fragebogen teilte sich in einen allgemeinen Teil (Alter, Geschlecht, Geburtsland, Fachsemester, ggfs. vorherige Ausbildung in einem Gesundheitsberuf) und einen speziellen Teil (Wissen hinsichtlich des eigenen Impfstatus, des Maserneliminationsziels der WHO, der Krankheitslast von Masern und der Akzeptanz von Impfempfehlungen).

Der Fragebogen ermittelte ebenso die persönliche Einstellung zur Masernimpfung unter den Medizinstudierenden. Folgende Statements bzw. Fragen wurden hierzu ausgewertet:

- Es ist wichtig, dass das Personal im Gesundheitswesen gegen Masern geimpft ist.

- Es ist wichtig, dass die Allgemeinbevölkerung gegen Masern geimpft ist.

- Würden Sie Ihr Kind nach den Empfehlungen der STIKO entsprechend gegen Masern impfen?

\section{Statistische Analysen}

Die tabellarische Auszählung der codierten Rohdaten erfolgte in Excel. Die statistische Auswertung der Daten wurde mit der Statistiksoftware BiAS für Windows 10.12 durchgeführt. Es wurden $95 \%$-Konfidenzintervalle (95\%-KI) berechnet. AuBerdem erfolgten die Signifikanzberechnungen mittels Chi-Quadrat-Vierfeldertafel-Test bzw. beim Vergleich der unterschiedlichen Untersuchungsjahre mit dem Chi-Quadrat-Kontingenztafeltest. Ein $p$-Wert $<0,05$ wurde als statistisch signifikant gewertet. Zusätzlich wurde ein Yates-Cochran-Test auf Trend durchgeführt, um signifikante Aufwärts- oder Abwärtstrends zu ermitteln.

\section{Ethikvotum}

Für die Studie lag ein positives Votum der Ethikkommission des Fachbereichs Medizin der Goethe-Universität vor (ReferenzNr. 30/14).

\section{Ergebnisse}

Die über den Zeitraum von Januar 2003 bis Dezember 2013 erhobenen Masernseroprävalenzdaten beziehen sich auf 9933 Untersuchungen. Von den 6456 Mitarbeitern (65\%) und 3477 Medizinstudierenden (35\%) waren $69,1 \%$ weiblich und 29,9\% männlich. Der Altersdurchschnitt der Teilnehmer betrug 29,4 Jahre. Bei den Mitarbeitern war der Altersdurchschnitt mit 32,6 Jahren höher als bei den Studierenden mit einem Altersdurchschnitt von 23,5 Jahren.

Von den 9933 Teilnehmern wiesen $85,7 \%$ (KI 85,0-86,4\%) einen positiven serologischen Befund auf, sodass eine Immunität angenommen wurde. Es wiesen $7,8 \%$ (KI 7,3-8,4\%) einen fraglichen Immunstatus bezüglich Masern auf und 6,3\% (KI 5,8-6,8\%) einen negativen Immunstatus. Der obere Wert des 95\%-Konfidenzintervalls des positiven Masernimmunstatus liegt im gesamten Untersuchungszeitraum unterhalb der von der WHO geforderten 95\%igen Immunitätsrate (siehe • Abb. 1). Zählt man den Anteil der Grenzwertigen hinzu, liegt der obere Wert des 95\%-Konfidenzintervalls im 
Bundesgesundheitsbl 2015 · 58:182-189 DOI 10.1007/s00103-014-2098-x

(c) Springer-Verlag Berlin Heidelberg 2014

\section{S. Petersen · H.F. Rabenau · A. Mankertz - D. Matysiak-Klose - I. Friedrichs · S. Wicker Immunität gegen Masern beim medizinischen Personal des Universitätsklinikums Frankfurt, 2003-2013}

\section{Zusammenfassung}

Hintergrund. Vor dem Hintergrund der angestrebten Masernelimination für das Jahr 2015 zeigt die aktuelle epidemiologische Situation in Deutschland, dass noch immer unzureichende Impfquoten in der Bevölkerung bestehen. In dieser Präeliminationsphase gewinnen nosokomiale Masern an Bedeutung. Ziel. Ziel unserer Studie war es, den Masernimmunstatus des medizinischen Personals und der Medizinstudierenden am Universitätsklinikum Frankfurt im Zeitverlauf festzustellen. Darüber hinaus sollte das Wissen der Medizinstudierenden beispielsweise hinsichtlich des Maserneliminationsziels der WHO und der Akzeptanz der Impfempfehlungen überprüft werden.

Methoden. Retrospektive Analyse der Masernseroprävalenz bei Mitarbeitern und Medizinstudierenden des Universitätsklinikums
Frankfurt nach Daten aus 11 Jahren. Medizinstudierende wurden im Frühjahr 2014 um Beantwortung eines anonymen Fragebogens zu ihrem Impfstatus, ihrem Wissen über Masern und der Akzeptanz von Impfempfehlungen gebeten.

Ergebnisse. Von den 9933 Untersuchten hatten $85,7 \%$ serologisch eine ausreichende Masernimmunität. Die Ergebnisse zeigten keine erkennbare Veränderung im Zeitverlauf. Es fand sich ein signifikanter Unterschied in den Immunitätsraten der bis 1970 und der nach 1970 geborenen Studienteilnehmer. Vor 1970 Geborene wiesen signifikant häufiger einen positiven Masernimmunstatus auf als Jüngere ( 96,4 vs. $83,3 \%, p<0,0001)$. Knapp 20\% der Medizinstudierenden kannten ihren eigenen Masernimpfstatus nicht und nur $70,7 \%$ wussten, dass sie zwei Masernimpfun- gen erhalten hatten. Lediglich 45,4\% der Medizinstudierenden kannten das Maserneliminationsziel der WHO, allerdings ließen sich 95\% der Studierenden anhand der Fragebogenerhebung als Impfbefürworter einteilen. Schlussfolgerung. Die erhobene Masernseroprävalenz der Studienpopulation erreicht die von der WHO angestrebte Immunitätsrate von $\geq 95 \%$ nicht. Das medizinische Personal war zu 87,4\%, die Medizinstudierenden zu 82,5\% seropositiv. Wenngleich eine hohe Akzeptanz der Medizinstudierenden hinsichtlich der Impfempfehlungen festgestellt wurde, ist das Wissen der Studierenden verbesserungswürdig.

\section{Schlüsselwörter}

Elimination · Impfung · Masern .

Medizinisches Personal

\section{Immunity against measles among healthcare personnel at the University Hospital Frankfurt, 2003-2013}

\section{Abstract}

Background. Despite aiming for the elimination of measles by 2015, the current epidemiological situation in Germany shows that there is still insufficient vaccination coverage among the population. During the present pre-elimination period, nosocomial measles are gaining in importance.

Aim. The purpose of our study was to determine the immune status of measles among healthcare personnel and medical students at the University Hospital Frankfurt. Moreover, the knowledge of study participants regarding the WHO's goal of the elimination of measles and the general attitude towards vaccination recommendations were investigated.

Methods. A retrospective study of measles seroprevalence was conducted among healthcare personnel and students at the University Hospital Frankfurt with an ob- servation period of 11 years. In spring 2014, medical students were asked to complete an anonymous questionnaire regarding vaccination status, knowledge of measles and acceptance of measles vaccination recommendations.

Results. In total, 9,933 serologies were evaluated and $85.7 \%$ of the participants had sufficient immunity against measles. Significant changes in immunity to measles over time were not observed. However, a significant difference in the immunity rate of participants born before 1970 and those born after 1970 was found. Participants born before 1970 significantly more often showed sufficient immunity against measles compared to those born later ( 96.4 vs. $83.3 \%, p<0.0001)$. Almost $20 \%$ of the medical students were not aware of their measles vaccination status and merely $70.7 \%$ indicated that they had received two measles vaccinations. Furthermore, only $45.4 \%$ of the medical students were familiar with the WHO's goal of eliminating measles by 2015 ; however $95 \%$ could be classified as vaccination-supporters on the basis of the questionnaire.

Conclusions. Overall, the immunity rate of measles determined by serology within the study population did not reach the WHO goal of $\geq 95 \%$, and this gap was even greater in group of medical students. Despite the medical students' positive attitude towards vaccination guidelines, the awareness in this field awaits improvement.

\section{Keywords}

Elimination · Vaccination - Measles .

Healthcare personnel
Jahr 2008/2009 sowie im Jahr 2012/2013 knapp über der geforderten 95\% Marke.

Die Mitarbeiter hatten insgesamt signifikant häufiger einen positiven Immunstatus $(87,4 \%)$ als die Medizinstudierenden $(82,5 \%)(p<0,0001)$, wohingegen zwischen Frauen und Männern kein signifikanter Unterschied beobachtet wurde.
Wenn man die Kollektive (Mitarbeiter und Medizinstudierende) allerdings vom abhängigen Faktor „vor oder nach 1970 geboren " bereinigt, besteht innerhalb der beiden Altersgruppen kein signifikanter Unterschied mehr in der jeweiligen $\mathrm{Ge}$ samtprävalenz ( $p=0,169$ bzw. $p=0,068$ bei den vor bzw. nach 1970 Geborenen). Im
Vergleich der Seroprävalenzdaten über die einzelnen Jahresgruppen seit 2003 zeigte sich, dass das medizinische Personal einen positiven Immunstatus zwischen 85,4 und $89,1 \%$ aufwies. Die Messungen zeigten keine erkennbare Tendenz im Zeitverlauf. Mittels des Yates-Cochran-Tests auf Trends ließ sich weder ein 


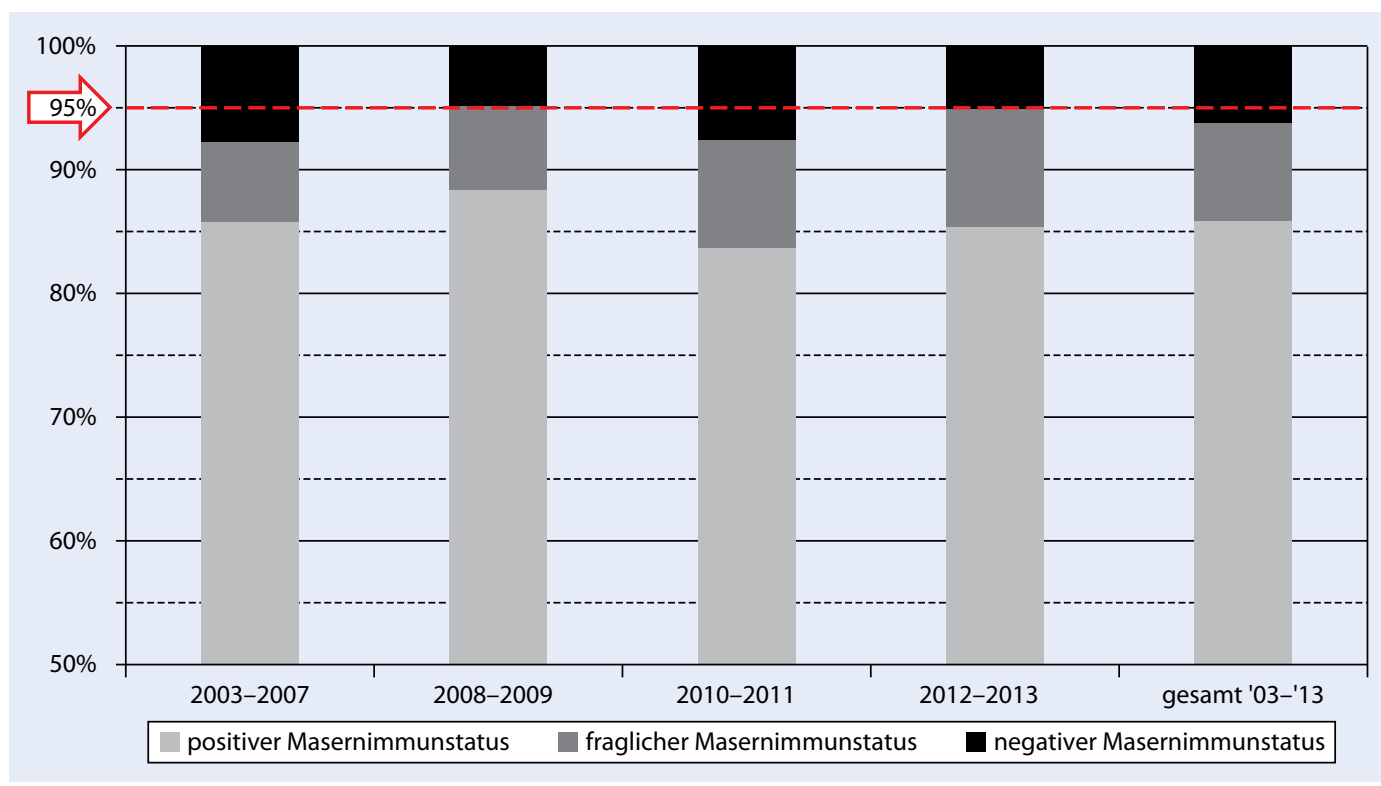

Abb. $1<$ Masernimmunstatus von medizinischen Beschäftigten und Medizinstudierenden des Universitätsklinikums Frankfurt im Zeitraum Januar 2003 bis Dezember $2013(n=9933)$ signifikanter Aufwärts- noch ein signifikanter Abwärtstrend ermitteln $(p=0,099)$, die Werte stagnierten auf hohem Niveau. Das Gleiche traf auf die Gruppe der Medizinstudierenden zu, wobei sich die Werte auf einem niedrigeren Niveau zwischen 77,1 und $86,3 \%$ bewegten (siehe $\bullet$ Abb. 2).

\section{Vergleich der Geburtsjahrgänge „bis 1970 Geborene" und „nach 1970 Geborene“}

Insgesamt 96,4\% der bis 1970 Geborenen wiesen einen positiven Masernimmunstatus auf, dagegen nur $83,3 \%$ bei den nach 1970 Geborenen $(p<0,0001)$ (siehe • Tab. 1). Zusammenfassend lässt sich feststellen, dass der Masernimmunstatus der Gesamtstudienpopulation nicht die angestrebte Immunitätsrate von mindestens 95\% erreicht. Eine größere Lücke ist insbesondere bei der Gruppe der Studierenden und den nach 1970 Geborenen vorhanden.

\section{Fragebogenerhebung}

Von den 1512 abgegebenen Fragebögen gingen 1421 auswertbar ausgefüllte Fragebögen (über $50 \%$ beantwortete Fragen) in die Analyse ein. Diese Zahl entspricht 50,6\% aller Medizinstudierenden der Goethe-Universität, die im Sommersemester 2014 immatrikuliert waren. Dabei handelte es sich um 919 Frauen $(64,7 \%)$ und 502 Männer (35,3\%), was dem Ge- schlechterverhältnis der Studierenden am Fachbereich Medizin entspricht (Frauen: 63,1\%, Männer: 36,9\%). Die Stichprobe spiegelt einen Querschnitt über alle Abschnitte des Medizinstudiums wider. Im Kollektiv befinden sich 613 Studierende im vorklinischen Abschnitt, 719 Studierende im klinischen Abschnitt, 72 Studierende im Praktischen Jahr und 17 Studierende direkt nach dem Staatsexamen. Für die Auswertung wurden die Studierenden in zwei Gruppen unterteilt: Gruppe 1: Studierende vor dem Physikum, Gruppe 2: Teilnehmer nach dem Physikum.

Insgesamt kannten 80,2\% der Studierenden (KI: 77,9-82,1\%) ihren Masernimpfstatus, Studierende nach dem Physikum kannten signifikant häufiger $(85,2 \%)$ ihren Impfstatus als Studierende vor dem Physikum $(73,4 \%)(p<0,0001)$. Insgesamt $70,7 \%$ gaben an, dass sie 2 Masernimpfungen erhalten hatten, 8,4\% hatten 1 Masernimpfung erhalten, 0,6\% waren nicht gegen Masern geimpft, $3 \%$ hatten Masern durchgemacht, 17,3\% konnten keine Angaben zu ihrem Impfstatus machen.

Das Maserneliminationsziel der WHO war 45,4\% der Medizinstudierenden bekannt (• Tab. 2) Es fand sich ein signifikanter Unterschied zwischen den Studierenden der Vorklinik (19,1\%) und den Studierenden des klinischen Studienabschnitts $(65,3 \%)(p<0,0001)$. Studierende mit einer vorherigen Ausbildung in einem Gesundheitsberuf kannten das Ma- serneliminationsziel signifikant häufiger ( $p=0,0015)$. Die Kenntnis des Maserneliminationsziels war unabhängig von den Parametern Geschlecht, Geburtsland und Bundesland.

Insgesamt $83,1 \%$ der Medizinstudierenden halten es für wichtig oder sehr wichtig, Masern zu eliminieren (- Abb. 3).

Hinsichtlich einer Analyse der Daten zur Einstellung gegenüber der Masernimpfung ließen sich die Probanden in drei Gruppen einteilen, die Unentschlossenen (4\%), die Impfbefürworter (95\%) und die Impfskeptiker/-gegner (1\%).

Insgesamt 71,6\% der Medizinstudierenden sprachen sich für eine verpflichtende Masernimpfung für alle Mitarbeiter im Gesundheitswesen aus; weitere $9,8 \%$ befürworteten eine verpflichtende Impfung für Beschäftigte, die abwehrgeschwächte Patienten betreuen; 9,4\% waren unentschlossen und 9,2\% lehnten eine verpflichtende Masernimpfung ab; es fand sich kein Unterschied in der Akzeptanz einer verpflichtenden Masernimpfung bei Studierenden vor bzw. nach dem Physikum.

\section{Diskussion}

An den von uns erhobenen serologischen Daten von fast 10.000 medizinischen Beschäftigten über einen Beobachtungszeitraum von 11 Jahren (Januar 2003-Dezember 2013) zeigte sich, dass das von der 


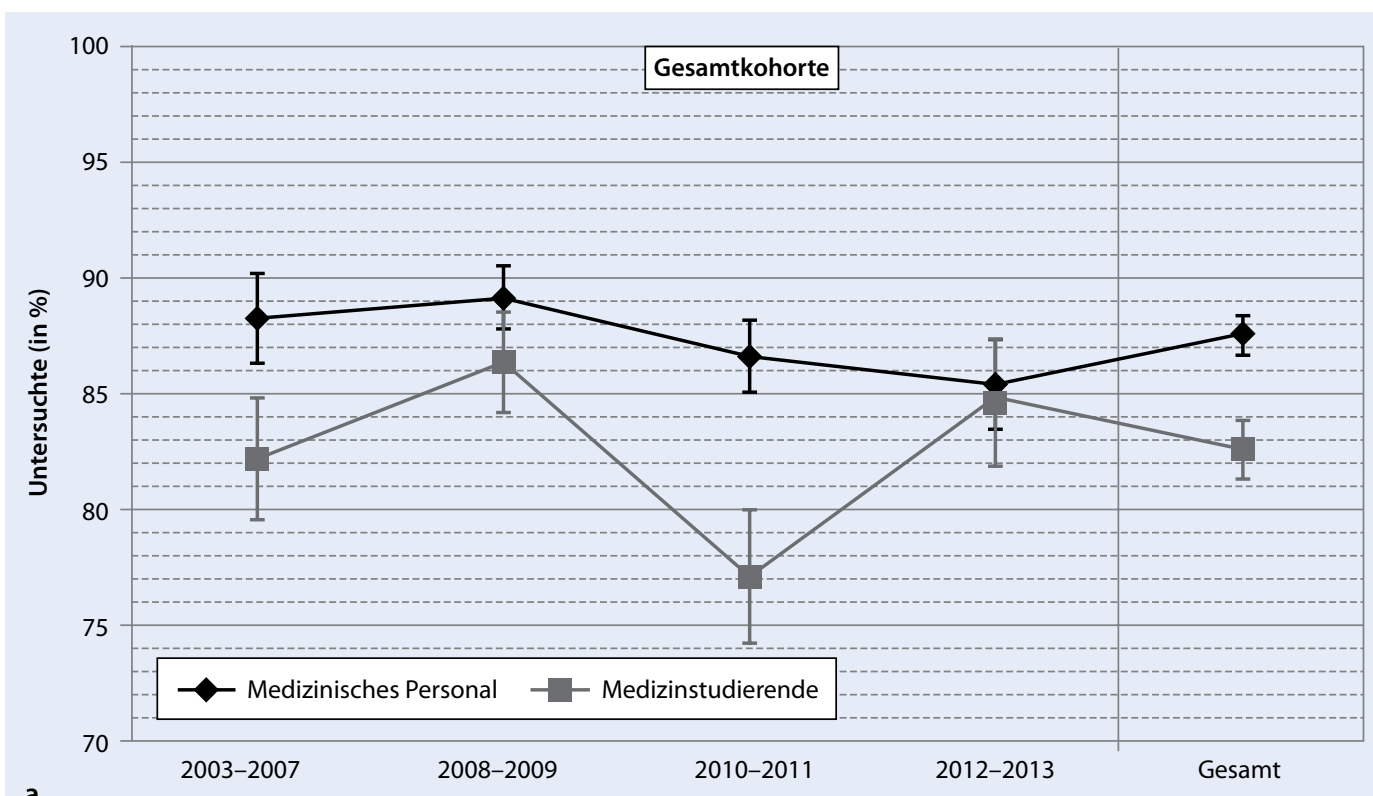

a

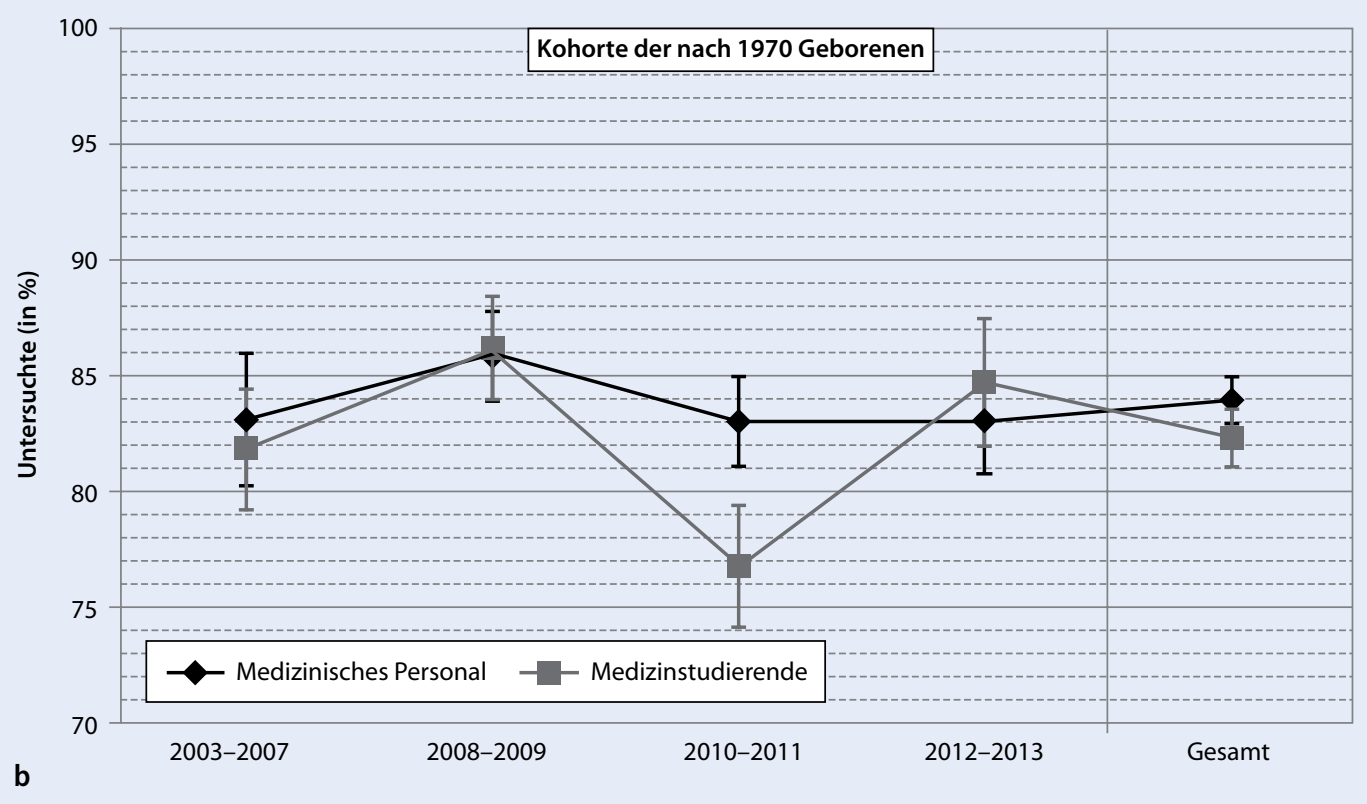

Abb. $2<$ Positiver Masernimmunstatus in Prozent (einschließlich 95\%-Konfidenzintervalle) im Vergleich für medizinische Beschäftigte und Medizinstudierende des Universitätsklinikums Frankfurt nach Untersuchungsjahren $(n=9933)$. a Gesamtkohorte. b Vergleich der jeweils nach 1970 geborenen medizinischen Beschäftigten $(n=4660)$ und Medizinstudierenden $(n=3429)$
WHO geforderte Eliminationskriterium einer $95 \%$ igen Masernimmunität des $\mathrm{Ge}$ samtkollektivs anhand der serologischen Werte nicht erreicht wurde und dass keine Verbesserungstendenz im Zeitverlauf besteht (siehe - Abb. 2). Es fand sich jedoch ein signifikanter Unterschied in den Immunitätsraten der bis und der nach 1970 geborenen Studienteilnehmer. Dem Vergleich liegt die Erwartung zugrunde, dass die bis 1970 geborenen Teilnehmer eine höhere Masernimmunität aufweisen, da Masernerkrankungen vor Einführung der Masernimpfung in den 1970er-Jahren wesentlich häufiger auftraten und die
Teilnehmer daher in einem erhöhten Maße eine natürliche Immunität aufweisen.

Insgesamt 96,4\% der bis 1970 geborenen Studienteilnehmer wiesen serologisch einen positiven Masernimmunitätsstatus auf, dagegen nur 83,3\% der nach $1970 \mathrm{Ge}$ borenen (siehe $\bullet$ Tab. 1). Dieses Ergebnis unterstreicht die Berechtigung der Masernimpfempfehlung der STIKO für die nach 1970 Geborenen, insbesondere für den medizinischen Bereich.

Aufgrund unzureichender Masernimpfquoten kommt es weiterhin zu Masernausbrüchen in der WHO-Region Europa [17-20], die länderübergreifend zu lang anhaltenden Transmissionsketten führen [21]. Einrichtungen des Gesundheitswesens spielen eine kritische Rolle bei der Übertragung von Masern [9, 13, 22, 23].

Bemerkenswerterweise besitzt die Hälfte der europäischen Länder keine spezifischen Masernimpfempfehlungen für medizinisches Personal [24]. Vor dem Hintergrund der hohen Anzahl an nosokomialen Maserninfektionen ist in diesen Ländern eine Überarbeitung der Impfempfehlungen dringend erforderlich. Aber auch in Deutschland besteht Handlungsbedarf. Hier bietet circa ein Drit- 


\begin{tabular}{|c|c|c|c|c|c|}
\hline \multirow{2}{*}{$\begin{array}{l}\text { Jahre der Untersu- } \\
\text { chung }\end{array}$} & \multicolumn{2}{|c|}{ Geburtsjahre } & \multicolumn{3}{|c|}{ Masernimmunstatus } \\
\hline & Anzahl & $\begin{array}{l}\text { Anteil am Ge- } \\
\text { samtkollektiv (\%) }\end{array}$ & Positiv & Fraglich & Negativ \\
\hline \multicolumn{6}{|c|}{ Geburtsjahre vor 1970} \\
\hline 2003-2007 & 447 & 21,9 & $\begin{array}{l}97,1 \% \\
(95,1-98,4)\end{array}$ & $\begin{array}{l}1,3 \% \\
(0,5-2,9)\end{array}$ & $\begin{array}{l}1,6 \% \\
(0,6-3,2)\end{array}$ \\
\hline 2008-2009 & 704 & 22,4 & $\begin{array}{l}95,6 \% \\
(93,8-97,0)\end{array}$ & $\begin{array}{l}2,6 \% \\
(1,5-4,0)\end{array}$ & $\begin{array}{l}1,8 \% \\
(1,0-3,1)\end{array}$ \\
\hline 2010-2011 & 483 & 18,0 & $\begin{array}{l}96,1 \% \\
(93,9-97,6)\end{array}$ & $\begin{array}{l}1,2 \% \\
(0,5-2,7)\end{array}$ & $\begin{array}{l}2,7 \% \\
(1,4-4,6)\end{array}$ \\
\hline 2012-2013 & 210 & 10,1 & $\begin{array}{l}98,6 \% \\
(95,9-99,7)\end{array}$ & $\begin{array}{l}0,5 \% \\
(0-2,6)\end{array}$ & $\begin{array}{l}0,9 \% \\
(0,1-3,4)\end{array}$ \\
\hline Gesamt & 1844 & 18,6 & $\begin{array}{l}96,4 \% \\
(95,5-97,2)\end{array}$ & $\begin{array}{l}1,7 \% \\
(1,1-2,4)\end{array}$ & $\begin{array}{l}1,9 \% \\
(1,3-2,6)\end{array}$ \\
\hline \multicolumn{6}{|c|}{ Geburtsjahre nach 1970} \\
\hline 2003-2007 & 1593 & 78,1 & $\begin{array}{l}82,5 \% \\
(80,5-84,3)\end{array}$ & $\begin{array}{l}7,8 \% \\
(6,6-9,3)\end{array}$ & $\begin{array}{l}9,7 \% \\
(8,3-11,2)\end{array}$ \\
\hline 2008-2009 & 2432 & 77,6 & $\begin{array}{l}86,1 \% \\
(84,6-87,4)\end{array}$ & $\begin{array}{l}8,1 \% \\
(7,1-9,3)\end{array}$ & $\begin{array}{l}5,8 \% \\
(4,9-6,8)\end{array}$ \\
\hline 2010-2011 & 2203 & 82,0 & $\begin{array}{l}80,6 \% \\
(79,0-82,4)\end{array}$ & $\begin{array}{l}10,5 \% \\
(9,2-11,8)\end{array}$ & $\begin{array}{l}8,9 \% \\
(7,8-10,2)\end{array}$ \\
\hline 2012-2013 & 1861 & 89,9 & $\begin{array}{l}83,7 \% \\
(81,9-85,3)\end{array}$ & $\begin{array}{l}10,7 \% \\
(9,3-12,2)\end{array}$ & $\begin{array}{l}5,6 \% \\
(4,6-6,8)\end{array}$ \\
\hline Gesamt & 8089 & 81,4 & $\begin{array}{l}83,3 \% \\
(82,5-84,2)\end{array}$ & $\begin{array}{l}9,3 \% \\
(8,7-10,0)\end{array}$ & $\begin{array}{l}7,4 \% \\
(6,8-8,0)\end{array}$ \\
\hline
\end{tabular}

tel der Betriebsärzte, die medizinisches Personal betreuen, die Masernimpfung nicht für das gesamte medizinische Personal, sondern nur in Teilbereichen (z. B. Gynäkologie, Pädiatrie) an [25]. So wundert es nicht, dass zahlreiche Seroprävalenzstudien bei medizinischen Beschäftigten in Europa fehlende Masernimmunitäten zwischen 1,2-8,5\% nachwiesen [13, 26-31]. Das Wissen um den Masernimmunstatus der medizinischen Beschäftigten ist daher von besonderer Bedeutung [1]. Das protektive Korrelat für Masern sind neutralisierende Antikörper der Titerstufe $\geq 1: 120$ [32, 33]. Da der Plaqueneutralisationstest für den Routineeinsatz nicht geeignet ist, werden zur Ermittlung des Schutzes der Nachweis von MasernIgG im ELISA-Test und/oder die dokumentierte zweimalige MMR-Impfung herangezogen. Dieses Vorgehen prognostiziert individuellen Schutz in einer guten Annäherung, wenn auch nicht mit absoluter Sicherheit, wie die bereits erwähnte Arbeit von Rosen et al. zeigt [15].

Nur wer Impfempfehlungen oder Public-Health-Ziele kennt, kann aktiv an deren Umsetzung mitarbeiten. Ärzte sind die wichtigste Informationsquelle für Patienten hinsichtlich der eigenen Impfentscheidung und der für ihre Kinder [34]. Daher war es von besonderem Wert, das Wissen unserer zukünftigen Ärzte in Bezug auf den eigenen Impfstatus, das WHO-Eliminationsziel sowie die Einstellung zur Masernimpfung zu ermitteln. Nahezu jeder 5. Medizinstudierende kannte seinen Masernimpfstatus nicht und nur circa $70 \%$ wussten, dass sie zwei Masernimpfungen erhalten hatten. Das Maserneliminationsziel der WHO war weniger als der Hälfte der Medizinstudierenden bekannt, von den Studierenden aus der Vorklinik kannte es noch nicht einmal jeder 5 . Studierende (• Tab. 2).

Wenngleich eine hohe Akzeptanz der Medizinstudierenden hinsichtlich der Impfempfehlungen belegt werden konnte und über $80 \%$ von ihnen eine verpflichtende Masernimpfung entweder für alle medizinischen Beschäftigten (71,6\%) bzw. eine verpflichtende Impfung für Beschäftigte, die abwehrgeschwächte Patienten betreuen $(9,8 \%)$, befürworten, kannte eine nicht unerhebliche Anzahl der Studierenden weder den eigenen Impfstatus
Tab. 2 Kenntnis des WHO-Maserneliminationsziels bei Medizinstudierenden ( $n=1421$; Erhebungszeitraum Frühjahr 2014)

\begin{tabular}{|c|c|c|}
\hline $\begin{array}{l}\text { Frage: „Ist Ihnen ein } \\
\text { Eliminationsziel für } \\
\text { Masern durch die } \\
\text { WHO bekannt?" }\end{array}$ & Ja & $\begin{array}{l}\text { Anteil in \% } \\
\text { (95\%-KI) }\end{array}$ \\
\hline Gesamt & 645 & $\begin{array}{l}45,4 \% \\
(42,7-48,0)\end{array}$ \\
\hline \multicolumn{3}{|l|}{ Studienabschnitt } \\
\hline Klinik & 528 & $\begin{array}{l}65,3 \% \\
(61,9-68,6)\end{array}$ \\
\hline Vorklinik & 117 & $\begin{array}{l}19,1 \% \\
(16,0-22,4)\end{array}$ \\
\hline \multicolumn{3}{|l|}{ Geschlecht } \\
\hline Weiblich & 418 & $\begin{array}{l}45,5 \% \\
(42,2-48,8)\end{array}$ \\
\hline Männlich & 227 & $\begin{array}{l}45,2 \% \\
(40,8-49,7) \\
\end{array}$ \\
\hline \multicolumn{3}{|l|}{ Geburtsort } \\
\hline Deutschland & 572 & $\begin{array}{l}45,7 \% \\
(42,9-48,5)\end{array}$ \\
\hline Ausland & 73 & $\begin{array}{l}43,2 \% \\
(35,6-51,0)\end{array}$ \\
\hline \multicolumn{3}{|c|}{ Aufgewachsen in welchem Bundesland } \\
\hline Neue Bundesländer & 133 & $\begin{array}{l}42,0 \% \\
(36,5-47,6)\end{array}$ \\
\hline Alte Bundesländer & 475 & $\begin{array}{l}46,5 \% \\
(43,4-49,6)\end{array}$ \\
\hline \multicolumn{3}{|c|}{ Ausbildungshintergrund } \\
\hline $\begin{array}{l}\text { Keine med. Vorbil- } \\
\text { dung }\end{array}$ & 535 & $\begin{array}{l}43,7 \% \\
(40,9-46,5)\end{array}$ \\
\hline $\begin{array}{l}\text { Mit Ausbildungshin- } \\
\text { tergrund }\end{array}$ & 110 & $\begin{array}{l}55,8 \% \\
(48,6-62,9)\end{array}$ \\
\hline
\end{tabular}

noch das WHO-Maserneliminationsziel. Hier wird deutlich, dass Impfungen als wichtige Präventionsmaßnahmen gegen Infektionskrankheiten in der Ausbildung von Medizinstudierenden eine größere Bedeutung erhalten müssen und der diesbezügliche Aufklärungs- und Schulungsbedarf groß ist.

Die vorliegende Studie hat Limitationen: Da die Datenauswertung anonymisiert erfolgte, konnten hierfür serologische Verlaufsproben einzelner Mitarbeiter nicht ausgeschlossen werden. Auch ist auf Basis der serologischen Untersuchungsergebnisse keine Differenzierung zwischen Durchimpfung und einer natürlich erworbenen Immunität möglich. Beim Fragebogen liegen Limitationen in der etwaigen Beantwortung im Sinne der sozialen Erwünschtheit und der fehlenden Nachprüfbarkeit der Angaben zum 


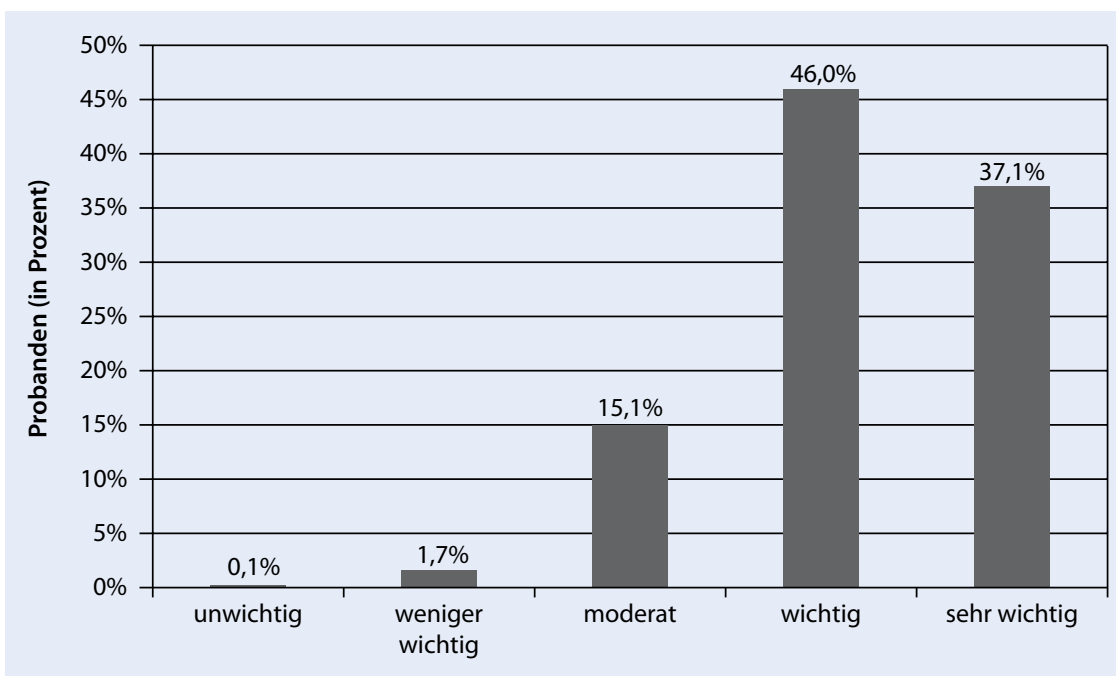

Abb. 3 - Wie wichtig ist das Maserneliminationsziel nach Meinung von Medizinstudierenden? ( $n=1421$; Erhebungszeitraum Frühjahr 2014)

Impfstatus. Es ist fraglich, inwieweit die Frankfurter Ergebnisse auf andere Krankenhäuser und Fakultäten übertragen werden können.

\section{Fazit}

Die aktuelle epidemiologische Situation in Deutschland und die Ergebnisse der hier vorgestellten Studie zur Masernseroprävalenz bei Mitarbeitern eines großen deutschen Universitätsklinikums zeigen, dass es zusätzlicher Anstrengungen bedarf, um die für 2015 geplante Maserneliminierung zu realisieren. Es können folgende Kernaussagen getroffen werden: - Lediglich $85,7 \%$ von fast 10.000 Studienteilnehmern zeigten serologisch eine ausreichende Masernimmunität, - die von der WHO geforderte 2-malige Masernimpfung mit einer Durchimpfung von mindestens $95 \%$ konnte über die Bestimmung der Seroprävalenz von IgG-Antikörpern nicht verifiziert werden,

- das Wissen der Studierenden über den eigenen Impfstatus und das Maserneliminationsziel der WHO ist verbesserungswürdig; die Ausbildung in Bezug auf Impfung als Präventionsmaßnahme muss dringend verbessert werden.

\section{Korrespondenzadresse}

\section{Prof. Dr. med. S. Wicker}

Betriebsärztlicher Dienst

Universitätsklinikum Frankfurt

Theodor-Stern-Kai 7, 60590 Frankfurt am Main

Sabine.Wicker@kgu.de

Danksagung. Die Autoren danken Frau Gabriele Bauer aus dem Institut für Medizinische Virologie für ihre Unterstützung bei der Zusammenstellung der serologischen Daten. Den Medizinstudierenden danken wir für das Ausfüllen des Fragebogens und die hilfreichen Anmerkungen zum Projekt.

\section{Einhaltung ethischer Richtlinien}

Interessenkonflikt. Die vertretenen Positionen entsprechen der persönlichen Einstellung der Autoren und repräsentieren nicht zwangsläufig die Position der medizinischen Organisationen oder Institutionen, denen sie angehören.

Prof. Wicker ist Mitglied der Ständigen Impfkommission (STIKO) und stellvertretende Vorsitzende der Nationalen Verifizierungskommission Masern/Röteln beim Robert Koch-Institut (NAVKO).

Dr. Matysiak-Klose leitet die Geschäftsstelle der NAVKO.

Prof. Mankertz leitet das Nationale Referenzzentrum für Masern, Mumps und Röteln (NRZ MMR) und ist ständiger Gast der NAVKO.

S. Petersen, Prof. Rabenau und Dr. Friedrichs erklären, dass keine Interessenkonflikte bestehen.

\section{Literatur}

1. Fiebelkorn AP, Seward JF, Orenstein WA (2013) A global perspective of vaccination of healthcare personnel against measles: systematic review. Vaccine 32:4823-4839
2. Fine PEM, Mulholland K (2013) Community immunity. In: Plotkin SA, Orenstein WA, Offit PA (Hrsg) Vaccines. Elsevier Saunders, Philadelphia, S 13951412

3. WHO Euro (2014) Eliminating Measles and Rubella. Framework for the verification process in the WHO European Region. http://www.euro. who.int/_data/assets/pdf_file/0009/247356/ Eliminating-measles-and-rubella-Framework-forthe-verification-process-in-the-WHO-EuropeanRegion.pdf?ua=1. Zugegriffen: 8. Aug. 2014

4. Davidkin I, Kontio M, Paunio M, Peltola H (2010) MMR vaccination and disease elimination: the Finnish experience. Expert Rev Vaccines 9:1045-1053

5. Robert Koch-Institut (2013) Aktuelle Epidemiologie und Impfquoten - wer erkrankt in Deutschland an Masern? Epidemiologisches Bulletin 48:485491

6. Matysiak-Klose D (2013) Hot Spot: Epidemiologie der Masern und Röteln in Deutschland und Europa. Bundesgesundheitsbl Gesundheitsforsch Gesundheitsschutz 56:1231-1237

7. Robert Koch-Institut (2014) https://survstat.rki.de/. Zugegriffen: 8. Aug. 2014

8. Botelho-Nevers E, Gautret P, Biellik R, Brouqui P (2012) Nosocomial transmission of measles. An updated review. Vaccine 30:3996-4001

9. Wicker S, Rabenau HF, Marckmann G, Gottschalk R (2013) Nosokomiale Maserninfektionen. Dtsch Med Wochenschr 138:2421-2425

10. Steingart RK, Thomas RA, Dykewicz AC, Redd CS (1999) Transmission of measles virus in healthcare settings during a communitywide outbreak. Infect Control Hosp Epidemiol 20:115-119

11. Chen SY, Anderson S, Kutty KP et al (2011) Health care-associated measles outbreak in the United States after an importation: challenges and economic impact. J Infect Dis 203:1517-1525

12. STIKO (2014) Empfehlungen der Ständigen Impfkommission (STIKO) am Robert Koch-Institut/ Stand August 2014. www.stiko.de. Zugegriffen: 26. Aug. 2014

13. Maltezou H, Wicker S (2013) Measles in health-care settings. Am J Infect Control 41:661-663

14. Robert Koch-Institut (2014) Masern - RKI Ratgeber für Ärzte, Stand 19.5.2014. http://www.rki.de/DE/ Content/Infekt/EpidBull/Merkblaetter/Ratgeber_ Masern.html\#doc2374536bodyText11. Zugegriffen: 8. Aug. 2014

15. Rosen JB, Rota JS, Hickman CJ et al (2014) Outbreak of measles among persons with prior evidence of immunity, New York City, 2011. Clin Infect Dis 58:1205-1210

16. Centers for Disease Control and Prevention (2011) Immunization of Health-Care Personnel - Recommendations of the Advisory Committee on Immunization Practices (ACIP). MMWR 60:1-46

17. Muscat M, Shefer A, Mamou MB et al (2014) The state of measles and rubella in the WHO European Region, 2013. Clin Microbiol Infect 20(Suppl 5):1218

18. Wicker S, Maltezou HC (2014) Vaccine preventable diseases in Europe: where do we stand? Expert Rev Vaccines 13:979-987

19. Mulholland EK, Griffiths UK, Biellik R (2012) Measles in the 21st century. N Engl J Med 366:17551757

20. Poland GA, Jacobson RM (2012) The re-emergence of measles in developed countries: time to develop the next-generation measles vaccines? Vaccine 30:103-104

21. Mankertz A, Mihneva Z, Gold H et al (2011) Spread of measles virus D4-Hamburg, Europe, 2008-2011. Emerg Infect Dis 17:1396-1401 
22. Sydnor E, Perl TM (2014) Healthcare providers as sources of vaccine-preventable diseases. Vaccine 32:4814-4822

23. Green M, Levin J, Michaels M et al (2012) Hospital-associated measles outbreak - Pennsylvania, March-April 2009. MMWR 61:30-32

24. Maltezou HC, Wicker S, Borg M et al (2011) Vaccination policies for health-care workers in acute health-care facilities in Europe. Vaccine 29:95579562

25. Betsch C, Wicker S (2014) Personal attitudes and misconceptions, not official recommendations guide occupational physicians' vaccination decisions. Vaccine 32:4478-4484

26. Celikbas A, Ergonul O, Aksaray S et al (2006) Measles, rubella, mumps, and varicella seroprevalence among health care workers in Turkey: is prevaccination screening cost-effective? Am J Infect Control 34:583-587

27. Ziegler E, Roth C, Wreghitt T (2003) Prevalence of measles susceptibility among health care workers in a UK hospital. Does the UK need to introduce a measles policy for its health care workers? Occup Med 53:398-402

28. Porru S, Campagna M, Arici C et al (2007) Susceptibility to varicella-zoster, measles, rosacea and mumps among health care workers in Northern Italy hospital. G Ital Med Lav Ergon 29(Suppl 3):407-409

29. Fernandez-Cano Ml, Armadans L et al (2012) Susceptibility to measles and varicella in healthcare workers in a tertiary hospital in Catalonia. Enferm Infect Microbiol Clin 30:184-188

30. Prato R, Tafuri S, Fortunato F, Martinelli D (2010) Vaccination in healthcare workers: an Italian perspective. Expert Rev Vaccines 9:277-283

31. Tafuri S, Martinelli D, Caputi G et al (2009) An audit of vaccination coverage among vaccination service workers in Puglia, Italy. Am J Infect Control 37:414-416

32. Plotkin SA (2008) Correlates of vaccine-induced immunity. Clin Infect Dis 47:401-409

33. Chen RT, Markowitz LE, Albrecht P et al (1990) Measles antibody: reevaluation of protective titers. J Infect Dis 162:1036-1042

34. Gaczkowska A, Mertens B, Reckendrees B, Wortberg S, Pott E (2013) Wissen, Einstellung und Verhalten zur Masernimpfung. Bundesgesundheitsbl Gesundheitsforsch Gesundheitsschutz 56:12701278 